\title{
Tofacitinib and Baricitinib Are Taken up by Different Uptake Mechanisms Determining the Efficacy of Both Drugs in RA
}

\author{
Jan Amrhein ${ }^{1}$, Susanne Drynda ${ }^{2}$, Lukas Schlatt ${ }^{3}$, Uwe Karst ${ }^{3}{ }^{\circledR}$, Christoph H. Lohmann ${ }^{2}$, \\ Giuliano Ciarimboli ${ }^{1}$ (D) and Jessica Bertrand ${ }^{2, *}$ \\ 1 Experimental Nephrology, Department of Internal Medicine D, University Hospital Münster, 48149 Münster, \\ Germany; jan.amrhein@web.de (J.A.); gciari@uni-muenster.de (G.C.) \\ 2 Department of Orthopedic Surgery, Otto-von-Guericke University, 39120 Magdeburg, Germany; \\ susanne.drynda@med.ovgu.de (S.D.); christoph.lohmann@med.ovgu.de (C.H.L.) \\ 3 Institute of Inorganic and Analytical Chemistry, University of Münster, 48149 Münster, Germany; \\ lukas.schlatt@uni-muenster.de (L.S.); uk@uni-muenster.de (U.K.) \\ * Correspondence: jessica.bertrand@med.ovgu.de; Tel.: +49-391-67-15804
}

Received: 27 August 2020; Accepted: 9 September 2020; Published: 10 September 2020

\begin{abstract}
Background: Rheumatoid arthritis (RA) is a systemic autoimmune disease in which synovial fibroblasts (SF) play a key role. Baricitinib and Tofacitinib both act intracellularly, blocking the ATP-binding side of JAK proteins and thereby the downstream signalling pathway via STAT-3. Therefore, we investigated the role of organic cation transporters (OCTs) in Baricitinib and Tofacitinib cellular transport. Methods: OCT expression was analysed in SF isolated from RA and osteoarthritis (OA) patients, as well as peripheral blood mononuclear cells. The interaction of Baricitinib and Tofacitinib with OCTs was investigated using quenching experiments. The intracellular accumulation of both drugs was quantified using LC/MS. Target inhibition for both drugs was tested using Western blot for phosphorylated JAK1 and STAT3 upon stimulation with IL-6. Results: MATE-1 expression increased in OASF compared to RASF. The other OCTs were not differentially expressed. The transport of Baricitinib was not OCT dependent. Tofacitinib; however, was exported from RASF in a MATE-1 dependent way. Tofacitinib and Baricitinib showed comparable inhibition of downstream signalling pathways. Conclusion: We observed different cellular uptake strategies for Baricitinib and Tofacitinib. Tofacitinib was exported out of healthy cells due to the increased expression of MATE1. This might make Tofacitinib the favourable drug.
\end{abstract}

Keywords: RA; Tofacitinib; Baricitinib; organic cation transporter; MATE1

\section{Introduction}

Rheumatoid arthritis (RA) is a systemic autoimmune disease that predominantly affects synovial joints, causing progressive polyarthritis, joint destruction and disability [1]. Synovial fibroblasts (SF) are key players in the development of RA [2]. In healthy synovial joints the synovium is formed of a few fibroblast layers, which mainly regulate the production of synovial fluid [3]. Fibroblasts also play a role in the inert immune system, carrying Toll-like receptors and being able to secrete cytokines $[4,5]$. In RA, fibroblasts evolve a tumour-like phenotype which transforms them to Rheumatoid Arthritis synovial fibroblasts (RASF) [6]. RASF acquire an aggressive phenotype with increased proliferation, loss of cell-cell contacts and joint invasiveness, where they secrete proinflammatory cytokines and interact with other immune and stroma cells to perpetuate the inflammatory reaction [2].

Cytokine signalling is a crucial driver of inflammation processes in RA. Besides tumour necrosis factor alpha (TNF- $\alpha$ ), Interleukin-6 (IL-6) mediates major inflammatory signalling pathways in RA [7]. 
Among other cytokines, it predominantly affects the JAK/STAT signalling. Janus kinases (JAKs) are non-receptor protein tyrosine kinases that consist of JAK1, JAK2, JAK3 and TYK2. IL-6 binds to the IL-6R $\alpha / g p 130$ complex that is linked to JAKs [8]. Upon binding of IL-6 to the receptor complex JAKs get phosphorylated by each other [9]. The cytosolic signal transducer and activator of transcription proteins (STATs) are able to bind to phosphorylated residues of the JAKs and get phosphorylated as well. They dimerize, translocate into the cell nucleus and act as transcription factors for the production of further proinflammatory cytokines, as well as cell differentiation and cell proliferation inducing factors [8].

Baricitinib and Tofacitinib are both relative new drugs, so called tyrosine kinase inhibitors (TKIs), approved and recommended by the European League Against Rheumatism (EULAR) for the treatment of RA $[10,11]$. These TKIs both act intracellularly, where they block the ATP-binding side of JAK proteins and thereby the downstream signalling pathway via STAT proteins. The binding of JAK inhibitors reduces cell differentiation, proliferation and production of proinflammatory cytokines [12,13]. Patients, that show an inadequate response to the disease-modifying drugs methotrexate (MTX) and/or biologics, may receive Tofacitinib or Baricitinib. Therefore, it is important to determine the optimal treatment options for these patients with regard to efficacy and safety of Tofacitinib and Baricitinib. To date, it is not known why different TKIs exert different effects in patients with the same disease. Differences in the uptake mechanism could explain these differences.

Around $40 \%$ of all orally administered drugs show cationic characteristics, and therefore need specific transport systems to penetrate nonpolar cell membranes to reach their intracellular target [14]. Previous studies showed that other TKIs like Imatinib and Saracatinib were dependent on polyspecific (meaning that they can accept structurally different substances as substrate) organic cation transporters (OCTs) to reach their intracellular target [15-17]. OCTs are part of the solute carrier (SLC) family [18]. This family includes the human organic cation transporters (hOCT1, hOCT2, hOCT3), the novel organic cation transporters (hOCTN1, hOCTN2), and the multidrug and toxin extrusion proteins (hMATE1, hMATE2k). Many of these OCTs share the same substrates, but every transporter has an individual substrate/inhibitor interaction profile. Whereas hMATE1, hMATE2k and hOCTN1 mediate a H+/organic cation (OC) antiport, hOCT1, hOCT2 and hOCT3 transport along the electrochemical gradient of their substrates. OCTs are widely expressed in different cells and are essential for the secretion of organic cations (OCs) in the liver and kidney [19]. Even though they transport mainly endogenous and exogenous OCs, interactions with zwitterions and anions have been reported [20,21].

As Tofacitinib and Baricitinib are established drugs for the treatment of RA, we investigated the uptake pathways of these drugs under RA relevant conditions focussing on their interaction with different OCTs and on their therapeutic efficiency in RASF.

\section{Results}

\subsection{Tofacitinib Could Be a Target for OCT Mediated Cellular Uptake}

Predictions on the pKa of Baricitinib and Tofacitinib, using the online tool Chemicalize of ChemAxon, showed that Baricitinib is not charged in neutral pH solutions (red box Figure 1B), whereas Tofacitinib is partially positively charged (red box, Figure 1A). Adjusting the $\mathrm{pH}$ to more acidic values, like in RA synovial fluid, supposes an increase of positively charged species of the two drugs. However, Baricitinib would largely stay uncharged, whereas Tofacitinib gets more positively charged making it a potential substrate for organic cation transporters. Therefore, we investigated the expression pattern of OCTs in OASF and RASF (Figure 1C). We observed no difference in the expression of $h O C T 1$ and hOCT3 between RA and OA synovial fibroblasts. hOCT2 was not detectable. $h O C T N 2$ was also only weakly expressed and no difference was seen between RASF and OASF. RASF, however, expressed significantly more $h O C T N 1(\mathrm{~F}(9,44)=12.06,95 \% \mathrm{CI}$ : 0.4480 to $1.157, p<0.0001)$. MATE-1 was lower expressed in RASFs compared to OASF $(\mathrm{F}(9,44)=12.06,95 \% \mathrm{CI}:-0.6278$ to $-0.01494, p=0.0358)$. This effect is even more pronounced in PBMCs from RA and OA patients (supplementary Figure S1). As 
both TKIs influence the IL-6 dependent pathways, we stimulated RASF with IL-6 and investigated the changes in the OCT expression pattern (Figure 1D). We observed an increase of $h M A T E-1$ expression, levelling out the differences between RASF and OASF. The other analysed OCTs were not changed in their expression.

\section{A Tofacitinib}
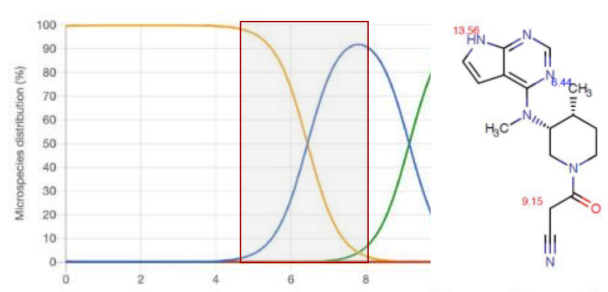

\section{B Baricitinib}

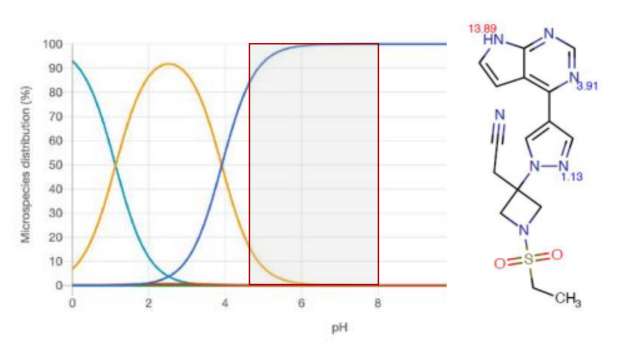

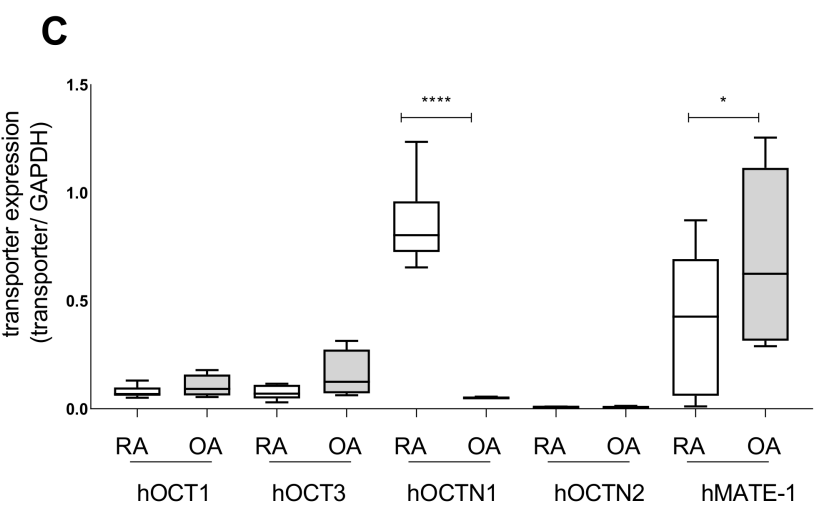

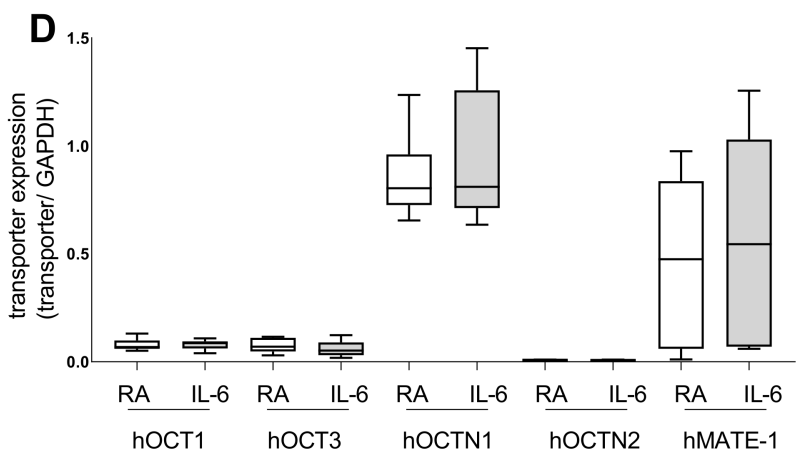

Figure 1. Tofacitinib could be a target for OCT mediated cellular uptake. (A,B) pKA prediction for Tofacitinib (A) and Baricitinib (B). The yellow curves indicate the presence of cationic form, the blue curves indicate the neutral form and the green curves of the anionic form of the substances at the given $\mathrm{pH}$-values. The red boxes indicate the physiological $\mathrm{pH}$-range. (C) Quantitative RT-PCR for the expression pattern of OCTs, and hMATE-1 from OASF and RASF. GAPDH was used as housekeeping gene for normalization. (D) Quantitative RT-PCR investigating the expression pattern of OCTs, and hMATE-1 in RASF after stimulation with $10 \mathrm{ng} / \mathrm{mL}$ IL-6 for $24 \mathrm{~h}$. GAPDH was used as housekeeping gene for normalization. Statistical analyses were performed using an ordinary one-way ANOVA and Sidak correction for multiple testing. ${ }^{*}: p<0.05 ;{ }^{* * * *}: p<0.0001$

\subsection{Baricitinib Uptake Is Not Transporter Dependent}

First, we investigated a potential interaction of Baricitinib with different OCTs using the ASP ${ }^{+}$ quenching method as a readout. We observed no significant interaction with any of the expressed OCTs in a physiological range of Baricitinib concentration (Figure 2A,B). To investigate a potential transporter dependent accumulation of Baricitinib in SF, we used OASF and RASF for LC/MS determination of intracellular Baricitinib concentrations. We observed no change in Baricitinib concentration neither depending on the temperature, nor depending on the disease (RASF vs OASF). We observed a higher intracellular Baricitinib concentration using $1 \mu \mathrm{M}$ (Figure 2C), compared to the approximate serum concentration of $0.15 \mu \mathrm{M}$ Baricitinib (Figure 2D). However, no temperature-dependent change in Baricitinib concentration was detected, indicating that the uncharged Baricitinib might be able to penetrate the cell membrane without active transport. 
A

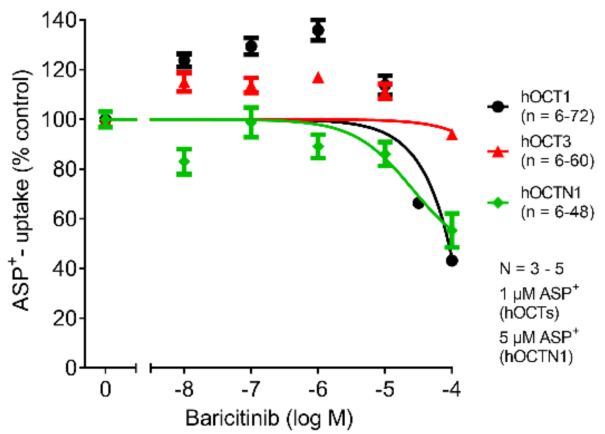

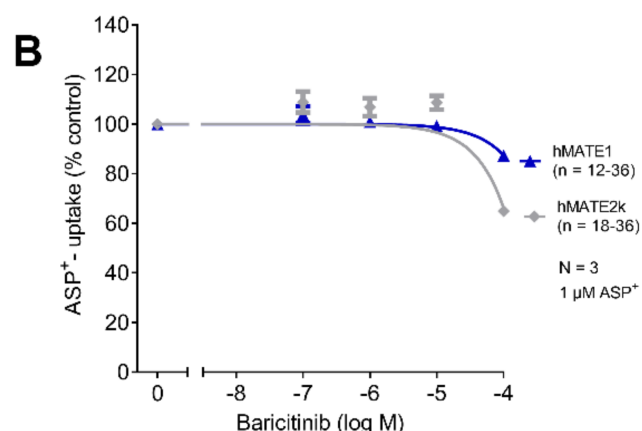

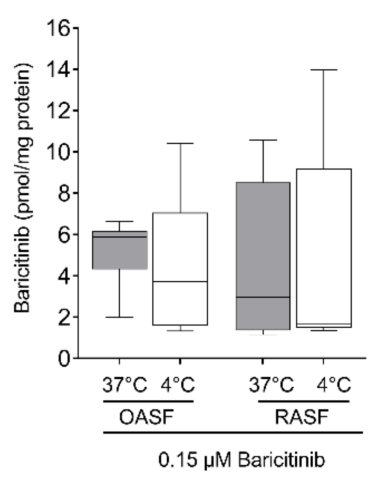

Figure 2. Baricitinib uptake is not transporter dependent. (A) $\mathrm{ASP}^{+}$determined apparent affinity of Baricitinib to hOCT1, hOCT3 and hOCTN1. No significant interaction was observed $(n=3-5)$. (B) $\mathrm{ASP}^{+}$determined affinity of hMATE1 and hMATE2k after intracellular acidification $(n=3)$. No significant interaction was observed. Data in $(\mathbf{A}, \mathbf{B})$ are presented as mean \pm SEM and fitted using a non-linear fit. (C) LC/MS measurement of temperature dependent Baricitinib $(1 \mu \mathrm{M})$ uptake in RASF and OASF $(\mathrm{F}(1.332,10.21)=1.570 ; p=0.2470 ; n=3)$. (D) LC/MS measurement of temperature dependent Baricitinib uptake $(0.15 \mu \mathrm{M})$ in RASF and OASF $(\mathrm{F}(2.071,16.56)=0.44, p=0.6578, n=3)$. Statistical analyses were performed using a RM ANOVA. No post-hoc testing was performed, as the ANOVA was not significant. Data in $(\mathbf{C}, \mathbf{D})$ are presented as box plot with whiskers indicating the min and max values, as well as the median.

\subsection{MATE-1 Mediates Tofacitinib Transport}

To test the affinity of Tofacitinib to different OCTs, we again performed the $\mathrm{ASP}^{+}$quenching assay. We observed no interaction of Tofacitinib with the OCTs (Figure 3A). Next, we investigated the interaction with MATE transporters using previous acidification. Interestingly, we found concentration-dependent inhibition of $\mathrm{ASP}^{+}$uptake by Tofacitinib in hMATE1 transfected HEK cells with an IC50 of $19.8 \mu \mathrm{M}$ (Figure 3B). To validate this finding, we used hMATE1-transfected HEK cells and observed a significant decrease in Tofacitinib intracellular accumulation at $37{ }^{\circ} \mathrm{C}$ $(\mathrm{F}(1.805,12.63)=20.71,95 \% \mathrm{CI}:-39.45$ to $-23.47, p<0.0001)$. This suggests a transporter mediated export of Tofacitinib via hMATE1 (Figure 3C). When investigating $1 \mu \mathrm{M}$ Tofacitinib concentration in OASF at $37^{\circ} \mathrm{C}$ and $4{ }^{\circ} \mathrm{C}$, we again observed significantly lower Tofacitinib concentrations at $37^{\circ} \mathrm{C}$ compared to $4{ }^{\circ} \mathrm{C}(1 \mu \mathrm{M})(\mathrm{F}(2.123,12.74)=10.15,95 \% \mathrm{CI}:-3.405$ to $-0.9026, p=0.0045)$ (Figure 3D). This effect was not as pronounced in RASF, which might be explained by the lower hMATE1 expression in RASF compared to OASF $(\mathrm{F}(2.123,12.74)=10.15,95 \% \mathrm{CI}$ : -2.549 to $0.6115, p=0.225)$. Using the approximate serum concentration of Tofacitinib for therapeutic use, we found the same pattern again, indicating that this effect is also present at low Tofacitinib concentrations (OASF: F $(1.969,15.75)=$ 6.925, 95\% CI: -2.118 to $-0.3189, p=0.012$ ) (Figure 3E). 
A

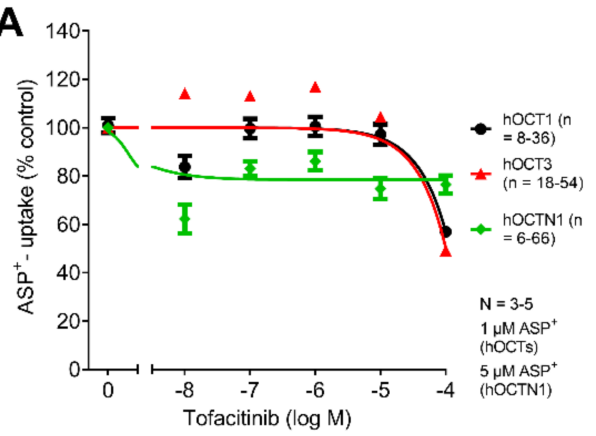

B

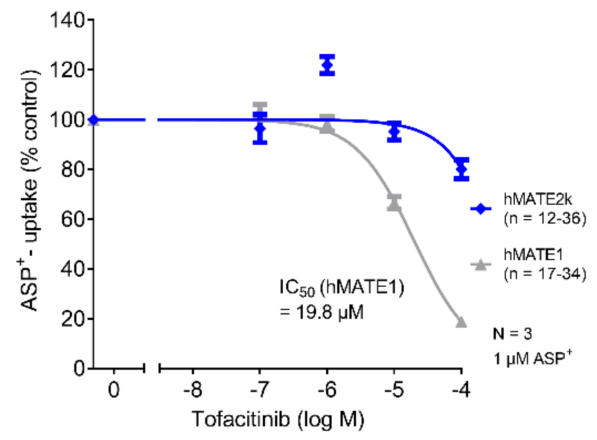

E

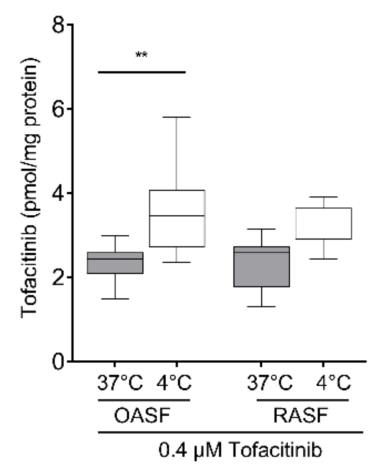

Figure 3. MATE-1 mediates Tofacitinib transport. (A) $\mathrm{ASP}^{+}$determined apparent affinity of Tofacitinib to hOCT1, hOCT3 and hOCTN1. No significant interaction was observed $(n=3-5)$. (B) ASP ${ }^{+}$ determined apparent affinity of hMATE1 and hMATE2k after intracellular acidification $(n=3)$. MATE-1 shows an apparent affinity IC50 $=19.8 \mu \mathrm{M}$ to MATE-1. Data in $(\mathbf{A}, \mathbf{B})$ are presented as mean \pm SEM and fitted using a non-linear fit. (C) LC/MS determined intracellular Tofacitinib concentration of HEK cells (control) and HEK cells overexpressing MATE- 1 at $37{ }^{\circ} \mathrm{C}$ and $4{ }^{\circ} \mathrm{C}(n=3)$. (D) LC/MS measurement of temperature dependent Baricitinib $(1 \mu \mathrm{M})$ uptake in RASF and OASF $(n=3)$. (E) LC/MS measurement of temperature dependent Baricitinib uptake $(0.4 \mu \mathrm{M})$ in RASF and OASF $(n=3)$. Statistical analyses were performed using a RM ANOVA and Sidak correction for multiple testing. Data in $(\mathbf{C}, \mathbf{D})$ are presented as box plot with whiskers indicating the min and max values, as well as the median. *: $p<0.05$; ${ }^{* *}: p<0.01 ;{ }^{* * *}: p<0.005$

\subsection{Tofacitinib and Baricitinib Showed Comparable Inhibition of IL-6-Induced STAT3-Phosphorylation}

As it was shown that Baricitinib and Tofacitinib are taken up by fibroblasts via different uptake mechanisms, we analysed the inhibition of JAK1 phosphorylation as well as the downstream target STAT3. Using $10 \mathrm{ng} / \mathrm{mL}$ IL-6 we activated the Jak1-STAT3 signalling pathway. We observed an increased time-dependent phosphorylation of Jak1 in RASF using IL-6 (untreated vs. 30 Min IL-6; 95\% CI: -0.24 to $-0.04 ; p=0.02$ ). Baricitinib did not influence phosphorylation JAK1 during the tested time course (Figure 4A). Tofacitinib, in contrast, time dependently inhibited the phosphorylation of JAk1 (Figure 4A). However, due to the very low amounts of pJAk1 detectable, these results did not reach statistical significance. OASF were less responsive towards IL-6 stimulation. They showed only a weak phosphorylation of Jak1 (untreated vs. $30 \mathrm{~min} \mathrm{IL}-695 \% \mathrm{CI}:-0.1$ to $0.002 ; p=0.05$ ), and no difference was observed using Baricitinib or Tofacitinib to inhibit the IL-6 induced phosphorylation (Figure 4B). Next, we investigated the phosphorylation of STAT3. As expected, $10 \mathrm{ng} / \mathrm{mL}$ IL-6 resulted in an increased time dependent phosphorylation of STAT3 in RASF (Time effect: $F(1.67,33.37)=$ 8.62; $p=0.002)$. Using either Baricitinib or Tofacitinib, this phosphorylation was inhibited, indicating an efficient inhibition of IL-6 induced signalling using both TKIs (Treatment effect: $F(2,24)=11.27$; $p=0.0004$ ). No significant difference was observed between both drugs (Figure 4C). We also investigated 
the efficacy of IL-6 blockade in OASF. There was less phosphorylation of STAT3 in unstimulated OASF, and these fibroblasts were also less responsive compared to RASF (Figure 4D). The treatment with either Tofacitinib or Baricitinib completely abolished the IL-6 induced phosphorylation of STAT3 (Treatment effect: $\mathrm{F}(1.179,9.432)=16.35 ; p=0.002)$. Again, no difference was observed between both TKIs.

A
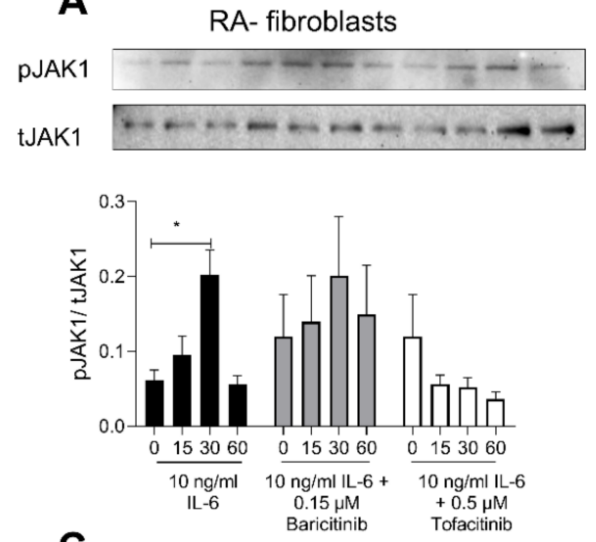

C
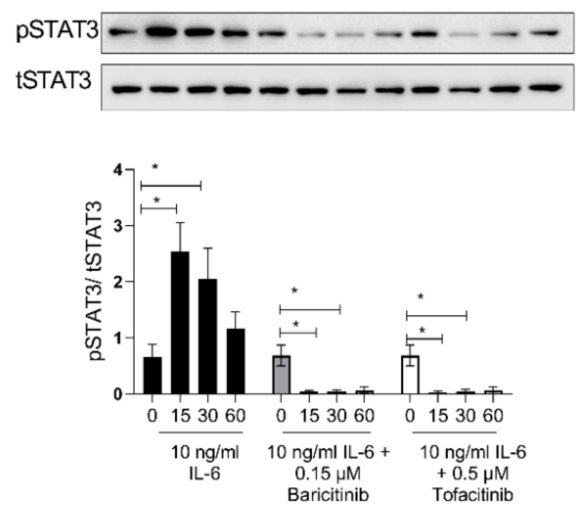

B
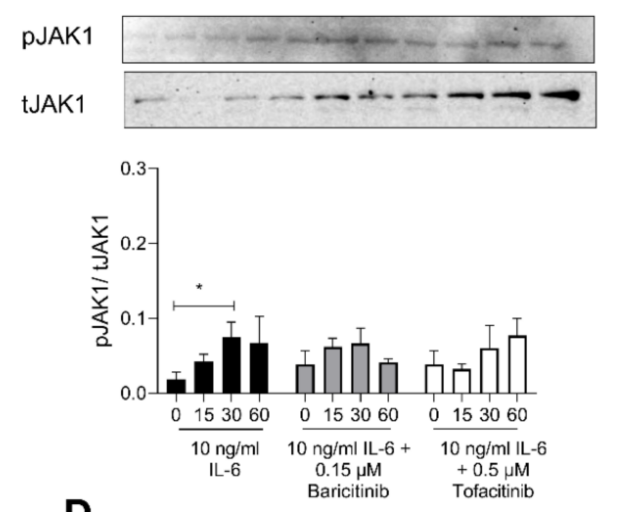

D

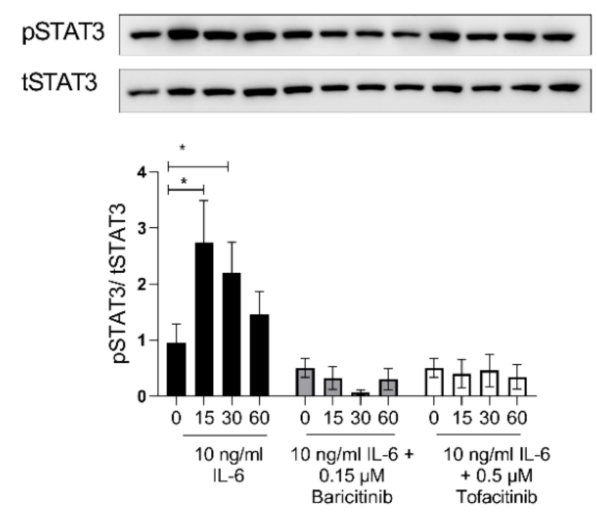

Figure 4. Tofacitinib and Baricitinib showed comparable inhibition of IL-6 induced STAT3phosphorylation. (A) Western blot for pJAK1 compared to total Jak1 in RASF stimulated for 0, 15,30 or $60 \mathrm{~min}$ with $10 \mathrm{ng} / \mathrm{mL}$ Il-6. Cells were also treated with $0.15 \mu \mathrm{M}$ Baricitinib or $0.5 \mu \mathrm{M}$ Tofacitinib. Quantification of at least 5 independent experiments are given in the graph underneath the blot. (B) Western blot for pJAK1 compared to total Jak1 in OASF stimulated with $10 \mathrm{ng} / \mathrm{mL} \mathrm{Il-6}$ and treated with $0.15 \mu \mathrm{M}$ Baricitinib or $0.5 \mu \mathrm{M}$ Tofacitinib. Quantification is given in the graph underneath the blot. (C) Western blot for pSTAT3 compared to total STAT3 in RASF stimulated for $0,15,30$ or 60 min with $10 \mathrm{ng} / \mathrm{mL}$ Il-6. Cells were also treated with $0.15 \mu \mathrm{M}$ Baricitinib or $0.5 \mu \mathrm{M}$ Tofacitinib. Quantification of at least five independent experiments is given in the graph underneath the blot. (D) Western blot for pSTAT3 compared to total STAT3 in OASF stimulated with $10 \mathrm{ng} / \mathrm{mL}$ Il- 6 and treated with $0.15 \mu \mathrm{M}$ Baricitinib or $0.5 \mu \mathrm{M}$ Tofacitinib. Quantification is given in the graph underneath the blot. A two-way RM-ANOVA was performed for statistical testing. Data are presented as mean values with SEM. A $p$-value $p \leq 0.05$ was considered to show statistical significance and is indicated by *.

\section{Discussion}

Both tested TKIs, Tofacitinib and Baricitinib, are approved as therapeutic drugs for the treatment of RA. Tofacitinib predominantly inhibits JAK1 and JAK3, and to a lesser degree JAK2 [13]. Baricitinib mainly inhibits JAK2, and acts only to a minor degree on the phosphorylation of JAK1 and JAK3 [12].

Tofacitinib was the first JAK inhibitor approved by the FDA in 2012 and subsequently by the EMA in 2017 for use in patients with moderate-to-severe RA at a dose of $5 \mathrm{mg}$ twice daily. Tofacitinib 
in combination with MTX is indicated for the treatment of moderate to severe active RA in adult patients who have responded inadequately to, or who are intolerant to, one or more disease-modifying antirheumatic drugs. Tofacitinib can be given as monotherapy in the case of intolerance to MTX, or when treatment with MTX is inappropriate. Baricitinib $2 \mathrm{mg}$ once daily (as monotherapy or combination therapy) was approved for RA patients with inadequate response to one or more tumour necrosis factor antagonist therapies in the US and for csDMARD-IR in Canada, while Baricitinib $2 \mathrm{mg}$ and $4 \mathrm{mg}$ (as monotherapy or combination therapy) were approved for RA patients with csDMARD-IR in Europe.

Variances in the clinical performance of both JAK inhibitors have been observed [22]. However, the reason for these differences are unknown given that they both TKIs target Janus Kinases resulting in a reduced STAT3 phosphorylation. However, each TKI has a different inhibitory profile against the different JAK isotypes [23,24]. This study aims to give an explanation for this observation by evaluating the intracellular uptake of both TKI into their targeted cells. A possible target in RA are synovial fibroblasts as they play an important role in the pathogenesis by contributing to joint destruction and producing cytokines [3]. RASF express several organic ion transporters which are capable of translocating TKIs, among them hOCTN1 and hMATE1, which have been previously reported to transport Saracatinib and Imatinib, respectively [14-17].

Baricitinib is not charged under physiological conditions, and therefore is no a target for organic cation transporters (Figure 1A). For this reason, we did not observe a transporter-mediated uptake in either the $\mathrm{ASP}^{+}$quenching tests (Figure 1C), nor the LC/MS detection of temperature dependent Baricitinib accumulation (Figure 2).

Investigating the role of organic cation transporters for the Tofacitinib accumulation in human RASF, we identified hMATE1 to predominantly mediate this transport (Figure 3C). Compared to OASF, hMATE1 expression is reduced in RASF (Figure 1C). We have previously shown that pro-inflammatory cytokines influence the MATE-1 expression [14]. RA is characterized by inflammatory processes that impact on various cellular activities [25]. Therefore, the influence of IL-6, which is also the main activator of JAK signalling, on OCT expression was analysed. IL-6 did not further impact on the expression of MATE-1 (Figure 1D).

Tofacitinib is charged under physiological pH-conditions (Figure 1B). Because transport of organic cations mediated by MATE-1 is $\mathrm{pH}$ dependent, we observed an export of Tofacitinib [21]. The synovial fluid in RA patients has been reported to exhibit an acidic $\mathrm{pH}$, under these conditions MATE- 1 is expected to mediate efflux of Tofacitinib $[25,26]$. To investigate the intracellular concentration of both TKIs we chose the maximum plasma concentration for Tofacitinib and Baricitinib for our experiment. The concentration for Baricitinib was described as $150 \mathrm{nM}$, and $400 \mathrm{nM}$ for Tofacitinib [27,28]. As expected, we did not observe a temperature-dependent increase of Baricitinib in fibroblasts (Figure 2D). This indicates that, due to its neutral charge, Baricitinib can penetrate the cell membrane without active transport. However, our data do not exclude that other transporters might contribute to the transport of Baricitinib into the cells. Tofacitinib in contrast is actively transported into fibroblasts. We found that MATE1 is the responsible transporter (Figure 3C). Interestingly, we found that OASF show a temperature-dependent lowering of Tofacitinib concentrations, indicating an active export of the drug from the cells (Figure 3D,E). This correlates with the increased MATE1 expression in OASF, which is reduced under inflammatory conditions. For this reason, in RASF, Tofacitinib is not exported.

We investigated the potency of both TKIs in inhibiting IL-6-induced JAK1 phosphorylation. As expected, Baricitinib did not inhibit JAK1 phosphorylation in RASF (Figure 4A). OASF were less responsive to IL-6 treatment (Figure 4B,D). Investigating the downstream transcription factor for IL-6 signalling, we observed no difference between Baricitinib and Tofacitinib, indicating that both TKIs are efficiently inhibiting the inflammatory response (Figure 4C).

The results from this study indicate that Tofacitinib might be exported from healthy cells, thereby not inhibiting the JAK pathway. Under disease conditions; however, Tofacitinib stays in the 
diseased cells and effectively inhibits the disease pathway. We observed no difference in inhibition of IL-6-induced inflammatory signalling for Tofacitinib and Baricitinib.

\section{Conclusions}

Thus, the differences in cellular uptake strategies for Baricitinib and Tofacitinib might explain the differences in clinical performance. Knowing that Tofacitinib is transported from healthy cells due to the increased expression of MATE1 might make it the more favourable drug.

\section{Materials and Methods}

\subsection{Cell Lines}

HEK293 cells (CRL-1573; American Type Culture Collection, Rochville, MD, USA) stably transfected with hOCT1 and hOCT3 were a kind gift of Prof. Koepsell (University of Würzburg) and grown with $600 \mu \mathrm{g} / \mathrm{mL}$ geneticin. HEK293 cells stably transfected with a hMATE1 plasmid (a gift by Dr. Yonezawa, Kyoto University Hospital, Japan Biochem. Pharmacol. 74: 359-371, 2007) were selected with $500 \mu \mathrm{g} / \mathrm{mL}$ hygromycin B. HEK293 cells transfected with cDNAs of hOCTN1 subcloned into a doxycycline-inducible pEBTetD plasmid vector were generously provided by Prof. Gründemann (University of Cologne, Cologne, Germany) [15,16] and selected with $3 \mathrm{mg} / \mathrm{L}$ puromycin. $24 \mathrm{~h}$ before starting experiments, hOCTN1 expression was induced by $1 \mathrm{mg} / \mathrm{L}$ doxycycline.

All cells were grown under standard conditions in Dulbecco's modified eagle medium (DMEM)—low glucose, containing $3.7 \mathrm{~g} / \mathrm{L} \mathrm{NaHCO} 3,1.0 \mathrm{~g} / \mathrm{L} \mathrm{D}$-glucose, $10 \%$ foetal calf serum, $100 \mathrm{U} / \mathrm{mL}$ penicillin/streptomycin, $1 \mathrm{mM}$ L-glutamine, gassed with $5 \% \mathrm{CO}_{2}$ at $37^{\circ} \mathrm{C}$.

\subsection{Synovial Fibroblasts (SF) Culture and Isolation}

SF were isolated from synovial tissue of rheumatoid arthritis (RA) $(n=10)$ and osteoarthritis (OA) $(n=10)$ patients undergoing joint replacement surgery. The Ethics Committee of the University of Magdeburg approved this study (IRR: 73/18), and all patients gave written consent prior to inclusion in the study. RA patients met the American College of Rheumatology criteria. Isolated fibroblasts were cultured under standard conditions for maximal eight passages. When indicated, RA synovial fibroblasts (RASF) were incubated with $10 \mathrm{ng} / \mathrm{mL}$ recombinant human IL-6 (R\&D).

\subsection{Peripheral Blood Mononuclear Cell (PBMC) Isolation and Cultivation}

Human PBMCs were isolated from RA and OA patients. The Ethics Committee of the University of Magdeburg approved this study (IRR: 73/18) and all patients gave written consent prior to inclusion in the study. In brief, $10 \mathrm{~mL}$ blood samples were centrifuged at $400 \times g$ in a Megafuge (Thermofisher Scientific, Berlin, Germany) for $10 \mathrm{~min}$ at room temperature. The cell pellet was resuspended in $\mathrm{PBS} / 0.1 \% \mathrm{BSA}$ and centrifuged at $300 \times \mathrm{g}$ at room temperature for $25 \mathrm{~min}$ without breaks using a Biocoll separating solution (Biochrom, Berlin, Germany). The generated lymphocyte ring was carefully taken off removed and washed two times twice with PBS/0.1\% BSA. Cells were cultured in Roswell Park Memorial Institute medium (RPMI 1640, Sigma-Aldrich, Taufkirchen, Germany) supplemented with $2 \mathrm{mM}$ L-glutamine, $10 \%$ foetal bovine serum, $1 \%$ penicillin/streptomycin solution at $37^{\circ} \mathrm{C}$ with $5 \%$ $\mathrm{CO}_{2}$.

\subsection{Apparent Affinities of Baricitinib and Tofacitinib for OCTs with 4-(4-(Dimethylamino)styryl)-N-} Methylpyridinium $\left(A S P^{+}\right)$

To investigate whether Baricitinib and Tofacitinib interact with OCTs (hOCT1, hOCT3, hOCTN1, hMATE1, hMATE2k), we used a dynamic cis-inhibition protocol of $\mathrm{ASP}^{+}$uptake, a known fluorescent substrate of all examined OCTs, with Baricitinib $\left(10^{-8}\right.$ to $\left.10^{-4} \mathrm{M}\right)$ and Tofacitinib $\left(10^{-8}\right.$ to $\left.10^{-4} \mathrm{M}\right)$, as described previously [14]. Briefly, HEK293 cells were seeded in 96-well plates and grown to $80-100 \%$ confluence. For experiments with hMATE-expressing cells, cellular $\mathrm{pH}$ was made acidic by $20 \mathrm{~min}$ 
preincubation with a modified ringer-like solution containing $\mathrm{NH}_{4} \mathrm{Cl}$ (in mM: $30 \mathrm{NH}_{4} \mathrm{Cl}, 115 \mathrm{NaCl}$, pH 7.4). Baricitinib and Tofacitinib (Hycultech, Beutelsbach, Germany) were dissolved in DMSO in ringer-like solution ( $\mathrm{RLS}=\mathrm{HCO}_{3}{ }^{-}$free Ringer-like solution containing (in mmol/L): $\mathrm{NaCl} 145$, $\mathrm{K}_{2} \mathrm{HPO}_{4} 1.6, \mathrm{KH}_{2} \mathrm{PO}_{4}$ 0.4, D-glucose 5, $\mathrm{MgCl}_{2}$, calcium gluconate 1.3 with pH adjusted to 7.4) to final concentrations in the range $10^{-4}-10^{-9} \mathrm{M}$. Fluorescence measurements were carried out with the TECAN@infinite F200 (Maennedorf, Switzerland). Transporter function was investigated measuring the slope of fluorescence emission (measured at $590 \mathrm{~nm}$ after excitation at $450 \mathrm{~nm}$ ) increase in the first $60 \mathrm{~s}$ after $\mathrm{ASP}^{+}$addition. $\mathrm{ASP}^{+}$uptake without potential inhibitor was set to $100 \%$.

\subsection{Quantification of Baricitinib and Tofacitinib Uptake by Liquid Chromatography Mass Spectrometry (LC/MS)}

HEK293 cells or RASF/OASF seeded into a six-well plate were grown to $80-100 \%$ confluence. Medium was removed and cells were incubated with Baricitinib and Tofacitinib in RLS at $37^{\circ} \mathrm{C}$ or $4{ }^{\circ} \mathrm{C}$ for $10 \mathrm{~min}$. After this incubation, cells were quickly washed with $1 \mathrm{~mL}$ ice-cold RLS, and then lysed with $300 \mu \mathrm{L} 0.1 \%$ formic acid. Cell lysates were incubated for $15 \mathrm{~min}$ in an ultrasound bath at $4{ }^{\circ} \mathrm{C}$ and centrifuged at $4700 \times g$ for $5 \mathrm{~min}$ at $4{ }^{\circ} \mathrm{C}$. $10 \mu \mathrm{L}$ internal standard (IS) of Baricitinib (Baricitinib-D5, Illkirch Graffenstaden, France) and Tofacitinib (Tofacitinib 13C3, Clearsynth, Mumbai, India) were added to the cell lysates for quantification. Acetonitrile (VWR, Radnor, Pennsylvania," USA) was added, mixed and centrifuged for $15 \mathrm{~min}$ with $16,200 \times g$ at $4{ }^{\circ} \mathrm{C} .200 \mu \mathrm{L}$ of the supernatant were diluted in distilled water to reach a final acetonitrile concentration of $12 \%$. The samples were frozen at $-80{ }^{\circ} \mathrm{C}$.

Quantification of Baricitinib and Tofacitinib concentration was performed using a high power liquid chromatography device (HPLC) (AdvanceTM UHPLC, Bruker Daltonik, Bremen, Germany) linked to a triple quadrupole mass spectrometer $\left(\mathrm{EVOQ}^{\circledR}\right.$ Elite triple quadrupole mass spectrometer, Bruker Daltonik, Bremen, Germany). A PAL HTC-xt autosampler (CTC Analytics AG, Zwingen, Switzerland) was used to inject $10 \mu \mathrm{l}$ of the sample to the HPLC. Analytes were separated on an AccucoreTM C18 HPLC column (50 mm $\times 3$ mm; $2.6 \mu \mathrm{m})$ (Thermo Scientific, Waltham, MA, USA). Amounts of $0.1 \%$ formic acid and acetonitrile were used as mobile phase A and $\mathrm{B}$.

Flow rate was set to $1 \mathrm{~mL} / \mathrm{min}$. Baricitinib and Tofacitinib had a specific retention time in HPLC. Ionization of the substances was realized using electrospray ionization (ESI) in a positive ionization mode. Spray voltage was set to $3500 \mathrm{~V}$, conus temperature to $350{ }^{\circ} \mathrm{C}$, gas flow to $60 \mathrm{AU}$, sample and cone temperature to $350{ }^{\circ} \mathrm{C}$. Exhaust gases were removed. First, Tofacitinib and Tofacitinib-IS, then Baricitinib and Baricitinib-IS were measured for $75 \mathrm{~ms}$ each. Full scan was applied to determine specific fission products of Baricitinib and Tofacitinib. Flow rate of the Cole-Parmer 74,900 single-syringe infusion pump (Vernon Hills, Illinois, USA) was set to $10 \mu \mathrm{L} / \mathrm{min}$, spray voltage to $4000 \mathrm{~V}$, gas flow to $10 \mathrm{AU}$, gas flow of the nebulizer to $10 \mathrm{AU}$ and temperature to $25^{\circ} \mathrm{C}$. Quantification took place in multiple reaction mode (MRM) (Table 1). Each sample was measured three times and the mean was calculated thereof.

Quantification was attained comparing the content of Baricitinib and Tofacitinib in the sample to the added IS (Figure 5). The result was normalized to the protein content determined in Bradford assay. Analyses were performed using MS Workstation (Bruker Daltonik, Bremen, Germany), MS Data review Version 8.2 (Bruker Daltonik, Bremen, Germany), Origin Pro 2016 (OriginLab, Northampton, MA, USA), GraphPad Prism 5 (GraphPad Software, San Diego, CA, USA) and Excel 2016. Method optimization and validation resulted in detection limits of Baricitinib and Tofacitinib of $0.9 \mathrm{ng} / \mathrm{mL}$ and $1.0 \mathrm{ng} / \mathrm{mL}$, and quantification limits of $3.0 \mathrm{ng} / \mathrm{mL}$ and $3.3 \mathrm{ng} / \mathrm{mL}$, respectively. 
Table 1. MRM transitions of Baricitinib, Tofacitinib, IS and collision energy. The grey-marked fragments had the highest measurement intensity and were used for quantification.

\begin{tabular}{ccc}
\hline Analyte & Transition $[\mathbf{m} / \mathbf{z}>\mathbf{m} / \mathbf{z}]$ & Collision Energy \\
\hline \multirow{2}{*}{ Tofacitinib } & $313>149$ & 28 \\
\cline { 2 - 3 } & $313>98$ & 31 \\
\cline { 2 - 3 } Tofacitinib-13C3 & $313>173$ & 37 \\
\cline { 2 - 3 } & $316>149$ & 28 \\
\cline { 2 - 3 } & $316>98$ & 31 \\
\hline \multirow{2}{*}{ Baricitinib } & $316>173$ & 37 \\
\cline { 2 - 3 } & $372>251$ & 26 \\
\hline \multirow{2}{*}{ Baricitinib-d5 } & $372>186$ & 31 \\
\hline & $377>251$ & 43 \\
\hline & $377>186$ & 26 \\
\hline
\end{tabular}

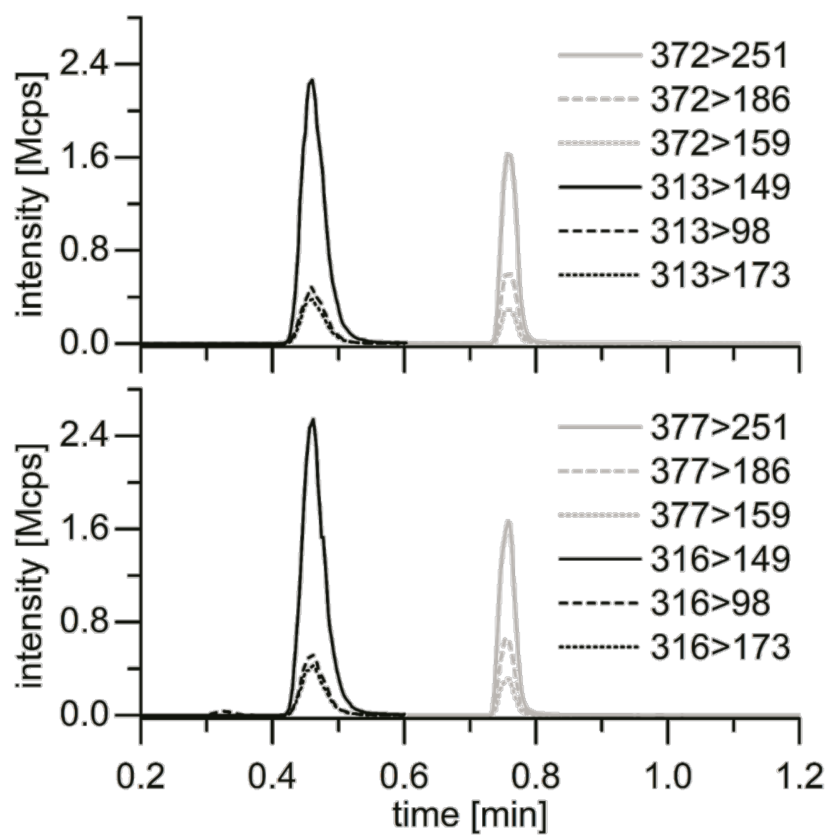

Figure 5. Transitions of Baricitinib and Tofacitinib (above) and IS (below). Tofacitinib is shown to the left in black, to the right Baricitinib in grey.

\subsection{Quantitative Real-Time PCR ( $q$ RT-PCR)}

RNA was isolated with the Qiagen RNeasy Midikit (Qiagen, Gilden, Germany) and Invitrogen Super Script III system was used for reverse transcription. qRT-PCR was performed using SYBR Green PCR Master Mix and the ABI PRISM 7900 Sequence Detection System (Applied Biosystems, Darmstadt, Germany) (primer pairs see supplementary Table S1). Gene expression is normalized to a semiquantitative standard curve and given in relation to the housekeeping gene GAPDH. 


\subsection{Western Blot Analysis}

For each RASF/OASF sample, a total of $1 \times 10^{6}$ cells were seeded in a $25 \mathrm{~cm}^{2}$ cell culture flask and the cells became adherent overnight. The cells were stimulated with $10 \mathrm{ng} / \mathrm{mL}$ IL-6 for 0, 15, 30 and 60 min with either Tofacitinib $(1 \mu \mathrm{M}, 0.4 \mu \mathrm{M})$ or Baricitinib $(1 \mu \mathrm{M}, 0.15 \mu \mathrm{M})$. Cells lysis was performed using NP-40- buffer and proteinase inhibitor cOmplete Ultra. Cell lysates were run on a 10\% SDS-PAGE and transferred to a PVDF membrane. Blocking was performed in $5 \%$ BSA solution. Primary antibody against phospho-Jak1 (\#74129), Jak-1 (\#3344), phosphor-Stat3 (\#9145), Stat-3 (\#30835), GAPDH (\#2118) diluted 1:1000 in 5\% BSA were incubated over night at $4{ }^{\circ} \mathrm{C}$. The secondary antibody was an HRP-conjugated anti-rabbit (\#7074) 1:8000 in 5\% BSA. All antibodies were bought from Cell Signaling (Danvers, MA, USA). Enhanced chemiluminescence (ECL) was used for Western blot detection, and quantification of bands was performed using ImageJ Software (U.S. National Institutes of Health, Bethesda, MD, USA, https://imagej.nih.gov/ij/, 1997-201).

\subsection{Statistical Analysis}

Data were analysed using GraphPad Prism, Version 5.0 (GraphPad Software, Inc., San Diego, CA, USA). To examine a statistical significance a two-way RM-ANOVA was performed. Sidak post-hoc multiple comparison test was performed to show intra individual significances. A $p$-value $p \leq 0.05$ was considered to show statistical significance. All experiments were repeated independently for at least three times.

Supplementary Materials: Supplementary materials can be found at http:/www.mdpi.com/1422-0067/21/18/ 6632/s1. Figure S1: Expression of organic cation transporters in PBMCs of OA and RA patients. Table S1: Primer for qRT-PCR.

Author Contributions: J.A. performed the experiments, S.D. isolated RNA from PBMCs and provided the patient blood samples, L.S. and U.K. performed the LC/MS experiments, C.H.L. provided the tissue samples for fibroblast isolation, G.C. and J.B. discussed and interpreted the data, J.B. wrote the manuscript. All authors have read and agreed to the published version of the manuscript.

Funding: Susanne Drynda, Giuliano Ciarimboli and Jessica Bertrand received funding from Pfizer for the project leading to this manuscript. This research was funded by an unrestricted grant provided by Pfizer Pharma GmbH, Forschungsförderung 2017 (IIR \#WI233723).

Acknowledgments: The authors acknowledge the excellent technical assistance of Carolin Schneider, Anja Schröder, Mandy Könnecke and Astrid Dirks. This project was supported by an unrestricted grant provided by Pfizer Pharma GmbH, Forschungsförderung 2017 (IIR \#WI233723).

Conflicts of Interest: The authors declare no conflict of interest. The funders had no role in the design of the study; in the collection, analyses, or interpretation of data; in the writing of the manuscript, or in the decision to publish the results.

\section{Abbreviations}

$\begin{array}{ll}\text { ANOVA } & \text { Analysis of variance } \\ \text { ASP } & \text { 4-(4-(dimethylamino)styryl)-N-methylpyridinium } \\ \text { AU } & \text { Arbitrary units } \\ \text { BSA } & \text { Bovine serum albumin } \\ \text { cDNA } & \text { complementary Deoxyribonucleic Acid } \\ \text { csDMARD-IR } & \text { conventional synthetic disease-modifying antirheumatic drug inadequate response } \\ \text { DMEM } & \text { Dulbecco's modified eagle medium } \\ \text { DMSO } & \text { Dimethyl sulfoxide } \\ \text { ECL } & \text { Enhanced chemiluminescence } \\ \text { EMA } & \text { European Medicines Agency } \\ \text { ESI } & \text { electrospray ionization } \\ \text { GAPDH } & \text { Glyceraldehyde 3-phosphate dehydrogenase } \\ \text { gp130 } & \text { Glycoprotein 130 } \\ \text { HEK 293 cells } & \text { Human embryonic kidney 293 cells } \\ \text { HPLC } & \text { High Performance Liquid Chromatography }\end{array}$




$\begin{array}{ll}\text { IL-6 } & \text { Interleukin-6 } \\ \text { IL-6Ra } & \text { Interleukin 6 Receptor Alpha } \\ \text { IS } & \text { Internal standrad } \\ \text { JAK } & \text { Janus Kinase } \\ \text { LC/MS } & \text { Liquid chromatography/mass spectrometry } \\ \text { MATE } & \text { multidrug and toxin extrusion } \\ \text { MRM } & \text { multiple reaction mode } \\ \text { MTX } & \text { methotrexate } \\ \text { NP-40 } & \text { Nonidet P-40 } \\ \text { OA } & \text { osteoarthrits } \\ \text { OC } & \text { Organic cation } \\ \text { OCT } & \text { Organic cation transporter } \\ \text { OCTN } & \text { Novel organic cation transporter } \\ \text { PBMCs } & \text { peripheral blood mononuclear cell } \\ \text { pKa } & \text { Ionization Constant } \\ \text { RA } & \text { Rheumatoid arthritis } \\ \text { RLS } & \text { ringer-like solution } \\ \text { RM-ANOVA } & \text { Repeated Measures Analysis of variance } \\ \text { RNA } & \text { Ribonucleic acid } \\ \text { RT-PCR } & \text { Reverse transcription polymerase chain reaction } \\ \text { SF } & \text { Synovial fibroblasts } \\ \text { STAT } & \text { Signal transducer and activator of transcription } \\ \text { TKI } & \text { Tyrosine kinase inhibitor } \\ \text { TNF-alpha } & \text { tumour necrosis factor alpha } \\ \text { TYK } & \text { Tyrosine kinase } \\ & \end{array}$

\section{References}

1. Smolen, J.S.; Aletaha, D.; McInnes, I.B. Rheumatoid arthritis. Lancet 2016, 388, 2023-2038. [CrossRef]

2. Huber, L.C.; Distler, O.; Tarner, I.; Gay, R.E.; Gay, S.; Pap, T. Synovial fibroblasts: Key players in rheumatoid arthritis. Rheumatology 2006, 45, 669-675. [CrossRef]

3. Ospelt, C. Synovial fibroblasts in 2017. RMD Open 2017, 3, e000471. [CrossRef] [PubMed]

4. Brentano, F.; Schorr, O.; Gay, R.E.; Gay, S.; Kyburz, D. RNA released from necrotic synovial fluid cells activates rheumatoid arthritis synovial fibroblasts via Toll-like receptor 3. Arthritis Rheum. 2005, 52, 2656-2665. [CrossRef] [PubMed]

5. Ospelt, C.; Brentano, F.; Rengel, Y.; Stanczyk, J.; Kolling, C.; Tak, P.P.; Gay, R.E.; Gay, S.; Kyburz, D. Overexpression of toll-like receptors 3 and 4 in synovial tissue from patients with early rheumatoid arthritis: Toll-like receptor expression in early and longstanding arthritis. Arthritis Rheum. 2008, 58, 3684-3692. [CrossRef] [PubMed]

6. Fassbender, H.G. Histomorphological basis of articular cartilage destruction in rheumatoid arthritis. Coll. Relat. Res. 1983, 3, 141-155. [CrossRef]

7. Wei, S.T.; Sun, Y.H.; Zong, S.H.; Xiang, Y.B. Serum Levels of IL-6 and TNF-alpha May Correlate with Activity and Severity of Rheumatoid Arthritis. Med. Sci. Monit. 2015, 21, 4030-4038. [CrossRef]

8. Malemud, C.J. The role of the JAK/STAT signal pathway in rheumatoid arthritis. Ther. Adv. Musculoskelet. Dis. 2018, 10, 117-127. [CrossRef]

9. Harrison, D.A. The Jak/STAT Pathway. Cold Spring Harb. Perspect. Biol. 2012, 4. [CrossRef]

10. Smolen, J.S.; Breedveld, F.C.; Burmester, G.R.; Bykerk, V.; Dougados, M.; Emery, P.; Kvien, T.K.; Navarro-Compan, M.V.; Oliver, S.; Schoels, M.; et al. Treating rheumatoid arthritis to target: 2014 update of the recommendations of an international task force. Ann. Rheum. Dis. 2016, 75, 3-15. [CrossRef]

11. Smolen, J.S.; Landewe, R.B.M.; Bijlsma, J.W.J.; Burmester, G.R.; Dougados, M.; Kerschbaumer, A.; McInnes, I.B.; Sepriano, A.; van Vollenhoven, R.F.; de Wit, M.; et al. EULAR recommendations for the management of rheumatoid arthritis with synthetic and biological disease-modifying antirheumatic drugs: 2019 update. Ann. Rheum. Dis. 2020, 79, 685-699. [CrossRef] 
12. Al-Salama, Z.T.; Scott, L.J. Baricitinib: A Review in Rheumatoid Arthritis. Drugs 2018, 78, 761-772. [CrossRef] [PubMed]

13. Dhillon, S. Tofacitinib: A Review in Rheumatoid Arthritis. Drugs 2017, 77, 1987-2001. [CrossRef] [PubMed]

14. Schmidt-Lauber, C.; Harrach, S.; Pap, T.; Fischer, M.; Victor, M.; Heitzmann, M.; Hansen, U.; Fobker, M.; Brand, S.M.; Sindic, A.; et al. Transport mechanisms and their pathology-induced regulation govern tyrosine kinase inhibitor delivery in rheumatoid arthritis. PLoS ONE 2012, 7, e52247. [CrossRef] [PubMed]

15. Harrach, S.; Barz, V.; Pap, T.; Pavenstadt, H.; Schlatter, E.; Edemir, B.; Distler, J.; Ciarimboli, G.; Bertrand, J. Notch Signaling Activity Determines Uptake and Biological Effect of Imatinib in Systemic Sclerosis Dermal Fibroblasts. J. Investig. Dermatol. 2019, 139, 439-447. [CrossRef] [PubMed]

16. Harrach, S.; Edemir, B.; Schmidt-Lauber, C.; Pap, T.; Bertrand, J.; Ciarimboli, G. Importance of the novel organic cation transporter 1 for tyrosine kinase inhibition by saracatinib in rheumatoid arthritis synovial fibroblasts. Sci. Rep. 2017, 7, 1258. [CrossRef] [PubMed]

17. Harrach, S.; Schmidt-Lauber, C.; Pap, T.; Pavenstadt, H.; Schlatter, E.; Schmidt, E.; Berdel, W.E.; Schulze, U.; Edemir, B.; Jeromin, S.; et al. MATE1 regulates cellular uptake and sensitivity to imatinib in CML patients. Blood Cancer J. 2016, 6, e470. [CrossRef]

18. Hediger, M.A.; Romero, M.F.; Peng, J.B.; Rolfs, A.; Takanaga, H.; Bruford, E.A. The ABCs of solute carriers: Physiological, pathological and therapeutic implications of human membrane transport proteinsIntroduction. Pflïgers Arch. 2004, 447, 465-468. [CrossRef]

19. Koepsell, H.; Endou, H. The SLC22 drug transporter family. Pflugers Arch. 2004, 447, 666-676. [CrossRef]

20. Takeda, M.; Khamdang, S.; Narikawa, S.; Kimura, H.; Kobayashi, Y.; Yamamoto, T.; Cha, S.H.; Sekine, T.; Endou, H. Human organic anion transporters and human organic cation transporters mediate renal antiviral transport. J. Pharmacol. Exp. Ther. 2002, 300, 918-924. [CrossRef]

21. Tanihara, Y.; Masuda, S.; Sato, T.; Katsura, T.; Ogawa, O.; Inui, K. Substrate specificity of MATE1 and MATE2-K, human multidrug and toxin extrusions/H(+)-organic cation antiporters. Biochem. Pharmacol. 2007, 74, 359-371. [CrossRef] [PubMed]

22. Westhovens, R. Clinical efficacy of new JAK inhibitors under development. Just more of the same? Rheumatology 2019, 58, i27-i33. [CrossRef] [PubMed]

23. O'Shea, J.J.; Kontzias, A.; Yamaoka, K.; Tanaka, Y.; Laurence, A. Janus kinase inhibitors in autoimmune diseases. Ann. Rheum. Dis. 2013, 72, 111-115. [CrossRef] [PubMed]

24. O'Shea, J.J.; Plenge, R. JAK and STAT signaling molecules in immunoregulation and immune-mediated disease. Immunity 2012, 36, 542-550. [CrossRef]

25. McInnes, I.B.; Schett, G. Cytokines in the pathogenesis of rheumatoid arthritis. Nat. Rev. Immunol. 2007, 7, 429-442. [CrossRef]

26. Farr, M.; Garvey, K.; Bold, A.M.; Kendall, M.J.; Bacon, P.A. Significance of the hydrogen ion concentration in synovial fluid in rheumatoid arthritis. Clin. Exp. Rheumatol. 1985, 3, 99-104.

27. Dowty, M.E.; Lin, J.; Ryder, T.F.; Wang, W.; Walker, G.S.; Vaz, A.; Chan, G.L.; Krishnaswami, S.; Prakash, C. The pharmacokinetics, metabolism, and clearance mechanisms of tofacitinib, a janus kinase inhibitor, in humans. Drug Metab. Dispos. 2014, 42, 759-773. [CrossRef]

28. Shi, J.G.; Chen, X.; Lee, F.; Emm, T.; Scherle, P.A.; Lo, Y.; Punwani, N.; Williams, W.V.; Yeleswaram, S. The pharmacokinetics, pharmacodynamics, and safety of baricitinib, an oral JAK 1/2 inhibitor, in healthy volunteers. J. Clin. Pharmacol. 2014, 54, 1354-1361. [CrossRef]

(C) 2020 by the authors. Licensee MDPI, Basel, Switzerland. This article is an open access article distributed under the terms and conditions of the Creative Commons Attribution (CC BY) license (http://creativecommons.org/licenses/by/4.0/). 